\title{
Improving the spatial resolution of XMM-Newton EPIC images by direct demodulation technique
}

\author{
Hua Feng ${ }^{1}$, Yong Chen ${ }^{2}$, S. N. Zhang ${ }^{3,4,5,2}$, F. J. $\mathrm{Lu}^{2}$, and T. P. Li ${ }^{3,2}$ \\ 1 Department of Engineering Physics, Tsinghua University, Beijing 100084, PR China \\ 2 Laboratory for Particle Astrophysics, Institute of High Energy Physics, CAS, Beijing 100039, PR China \\ 3 Physics Department and Center for Astrophysics, Tsinghua University, Beijing 100084, PR China \\ ${ }^{4}$ Physics Department, University of Alabama in Huntsville, Huntsville, AL 35899, USA \\ 5 Space Science Laboratory, NASA Marshall Space Flight Center, SD50, Huntsville, AL 35812, USA
}

Received 18 November 2002 / Accepted 24 February 2003

\begin{abstract}
We have applied the direct demodulation technique to XMM-Newton EPIC images to achieve a higher spatial resolution; the restored images are compared with Chandra images. With the improved images we can extract spectra of fine structures in XMM-Newton data.
\end{abstract}

Key words. techniques: high angular resolution, techniques: image processing

\section{Introduction}

The XMM-Newton Observatory is a cornerstone mission of the ESA Horizon 2000 programme. XMM carries three X-ray focusing telescopes with the largest effective area than ever. In particular EPIC pn has an effective area about 3 times of a single MOS. However pn's wide point spread function (PSF) and large pixel size cause worse spatial resolution than MOS. In Table 1 we list the spatial resolutions and effective areas of XMM and Chandra. Though Chandra's spatial resolution is the highest, its effective area is much smaller than XMM. In the study of faint extended sources, Chandra can reveal fine structures but can not record enough photons to obtain a good spectrum for a small region. For XMM, though the photon numbers are much larger, we can not make a spectrum of a small region whose size is comparable to or smaller than the size of the PSF.

The direct demodulation (DD) technique has been proved an effective technique for image restoring, by solving the convolution equation (Eq. (1)) through iterations with constraints of physical conditions ( $\mathrm{Li} \& \mathrm{Wu} 1994$ )

$P S F * f=d$

where $d$ is the observed image and $f$ is the real distribution of the source in the sky. Finding $f$ by solving Eq. (1) is a typical ill-posed inversion problem. Conventional methods widely used to solve this problem in astronomy can be divided into two classes, cross-correlation techniques and

Send offprint requests to: Hua Feng,

e-mail: fenghua01@mails.tsinghua.edu.cn
Table 1. Spatial resolutions and effective areas of XMM \& Chandra.

\begin{tabular}{lrrr}
\hline \hline Instrument & $F W H M^{\mathrm{a}}\left[{ }^{\prime \prime}\right]$ & $H E W^{\mathrm{b}}\left[{ }^{\prime \prime}\right]$ & $\mathrm{Ae} @ 1 \mathrm{keV}^{\mathrm{c}}$ \\
\hline XMM MOS & 5 & 14 & $400^{\mathrm{d}}$ \\
XMM pn & 6 & 15 & 1200 \\
Chandra ACIS-I & 0.2 & 0.5 & 400 \\
\hline
\end{tabular}

a Full Width at Half Maximum.

b Half Energy Width.

c Approximate mirror effective areas with detector responses at $1 \mathrm{keV}$ in units of $\mathrm{cm}^{2}$.

d single MOS module.

statistical reconstruction techniques. The generally used statistical reconstruction techniques are maximum-entropy method, maximum-likelihood method, etc. These two classes of conventional methods have no significant difference in their inversion results. With either class of methods we can get the resultant resolution approaching the intrinsic resolution $(\Delta)$. The DD technique is much more effective to this kind of inversion problem with a resultant resolution much smaller than $\Delta$. Its processes can be simplified to three steps. Firstly we should convert Eq. (1) to a correlation equation (Eq. (2)). Thus we obtain a symmetric positive-definite matrix $P^{*}$

$P^{*} f=c$

where $P^{*}=P S F^{T} P S F$ and $c=P S F^{T} d$. Then the background of the image should be estimated either by the CLEAN 
technique (Högbom 1974) or from a region in the image without bright sources. In the end, we solve Eq. (2) with a certain iteration method (In Eq. (3). IM means a certain iteration method, such as the Gauss-Seidel iteration)

$f^{(l)}=I M\left(f^{(l-1)}\right)$

with a constraint condition (Eq. (4)).

if $f_{i}^{(l)}<b_{i}$ then $f_{i}^{(l)}=b_{i}$

where $b$ is the estimated background and $i$ is the pixel index. It has been proved that the above process is stable and will converge to the global optimum solution (Li 1997). Consequently this technique has been applied successfully to imaging analysis of many missions and different kinds of instruments, e.g., EXOSAT/ME (Lu et al. 1996), HEAO1-A4 (Lu et al. 1996), CGRO/COMPTEL (Zhang et al. 1997, 1998), RXTE/ASM (Chen et al. 2000), ROSAT/PSPC (Chen et al. 1997; Lu et al. 2001), coded mask telescope (Li 1995) and rotation modulation telescope (Chen et al. 1998).

In this paper we apply the DD technique to make a series of restored sub-images in different energy channel each. Then a restored image in a certain energy band can be produced by adding all sub-images in this energy band; an energy spectrum can then be made by counting the photons in each sub-image. This is the first time the DD technique has been applied for obtaining spectral information from restored images.

\section{Image restoring processes}

Because the PSF of XMM is energy dependent, the image restoring should be done in different energy channels. The PSF of XMM can be described as a King function. The best fitted King profile parameters are available in the latest in-flight calibration documents from the web (http://xmm.vilspa.esa.es/external/xmm_sw_cal/ calib_frame.shtml). The steps to obtaining a restored image or a spectrum from a restored image are as following.

(a) Make a spectrum in the interested area;

(b) Divide the spectrum into a number of new channel bounds with approximately the same photons in each channel;

(c) Produce a series of raw images with the energy channel bounds calculated in (b);

(d) Calculate the weighted average energy for every channel;

(e) Produce the PSF for the average energy and the off-axis angle in each channel;

(f) Restore the image with the DD technique and get a restored sub-image in each channel;

(g) Get a restored image by adding all energy dependent restored sub-images;

(h) Select a region of interest in the restored image produced from $(\mathrm{g})$ and get the spectrum of this region by counting the total photons in this region from each restored sub-image. In this way we can get a restored image and a spectrum in the region of interest.

Because the DD process is non-linear, the direct error estimation is difficult to make; the error depends on the shape of
PSF, the iteration steps, the total counts of observation, the distribution of the source, the background level and so on. We therefore apply the bootstrap method. We first use Monte-Carlo simulations to evaluate if the bootstrapped error is reliable. For an assumed image, we use Poisson sampling to generate many "observations". Then we restore all the "observations" by the DD technique to produce the distribution of the counts in some region; we call this DD-distribution. Finally we select any one "observation" as our bootstrapping seed image and make many bootstrapped images from it. After restoring these bootstrapped images and counting the photons in the same region, a bootstrap-distribution is calculated. We find that the DD-distribution and the bootstrap-distribution are both normal distributions with almost the same parameters. So a standard deviation from bootstrapping can be used as the error produced by the DD process.

We therefore add these following steps for the error estimation:

(i) Bootstrap $N$ images from each raw sub-image ( $N=$ 100 200 depending on the total counts in the raw image);

(j) Repeat (f) and (h);

(k) The standard deviations of the photon counts in the interested region of each bootstrapped image can be taken as the statistical errors of this region in this energy channel.

The bootstrapped deviation includes both Poisson fluctuation and the error introduced by DD processes, hence the standard deviation calculated from $(\mathrm{k})$ should be regarded as the finial statistical error.

\section{Restored images and spectra}

We apply the above processes on several sources which have been observed with both XMM and Chandra, e.g., M 87, Abell 1795 and PKS 0745-191. From the Chandra observations of these three sources, we can see that M 87 has the finest structure, PKS 0745-191 has the largest structure scale and Abell 1795 is in between. For M 87, we can see the nucleus and the brightest knot from both EPIC MOS and pn in the raw images. But for EPIC pn, it is difficult to separate the nucleus and the brightest knot, because their distance is smaller than the $H E W$ of EPIC pn. For Abell 1795 and PKS 0745-191, from XMM EPIC pn raw images we can not see any clear structures that showed in Chandra images. After the DD restoring, we can separate clearly the nucleus and the brightest knot of M 87 with pn data, and the structures in Abell 1795 and PKS 0745191 are also revealed. The XMM raw images, restored images and Chandra images are all shown from Figs. 1 to 3 for comparison. To evaluate the resolution of the restored images, we smooth the Chandra images with a Gaussian function with an $\sigma$ of 1.2 arcsec. From the figures we can see that the restored images have approximately the same resolution as the smoothed Chandra images. This means that the restored images have a resolution of about $3 \operatorname{arcsec}(H E W)$, about 5 times better than XMM EPIC raw images.

The spectra of M 87's nucleus and brightest knot from the restored images are shown in Fig. 4, which can be well fitted by an absorbed power-law model consistent with the previous results of Chandra (Wilson \& Yang 2002) and 

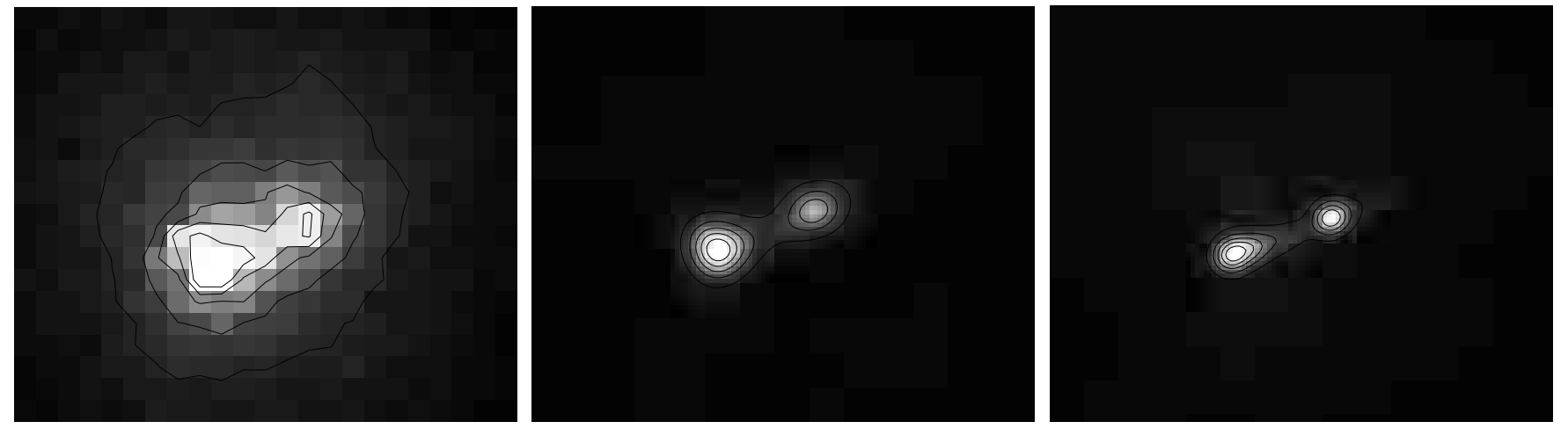

Fig. 1. M 87 images. left: XMM pn raw image $(0.2 \sim 6 \mathrm{keV}$, pixel size $=2.5 \operatorname{arcsec})$; center: restored image from left (pixel size $=0.5$ arcsec); right: Chandra ACIS-I image smoothed by a Gaussian filter with the $\sigma$ of $1.2 \operatorname{arcsec}$ (whole energy band).
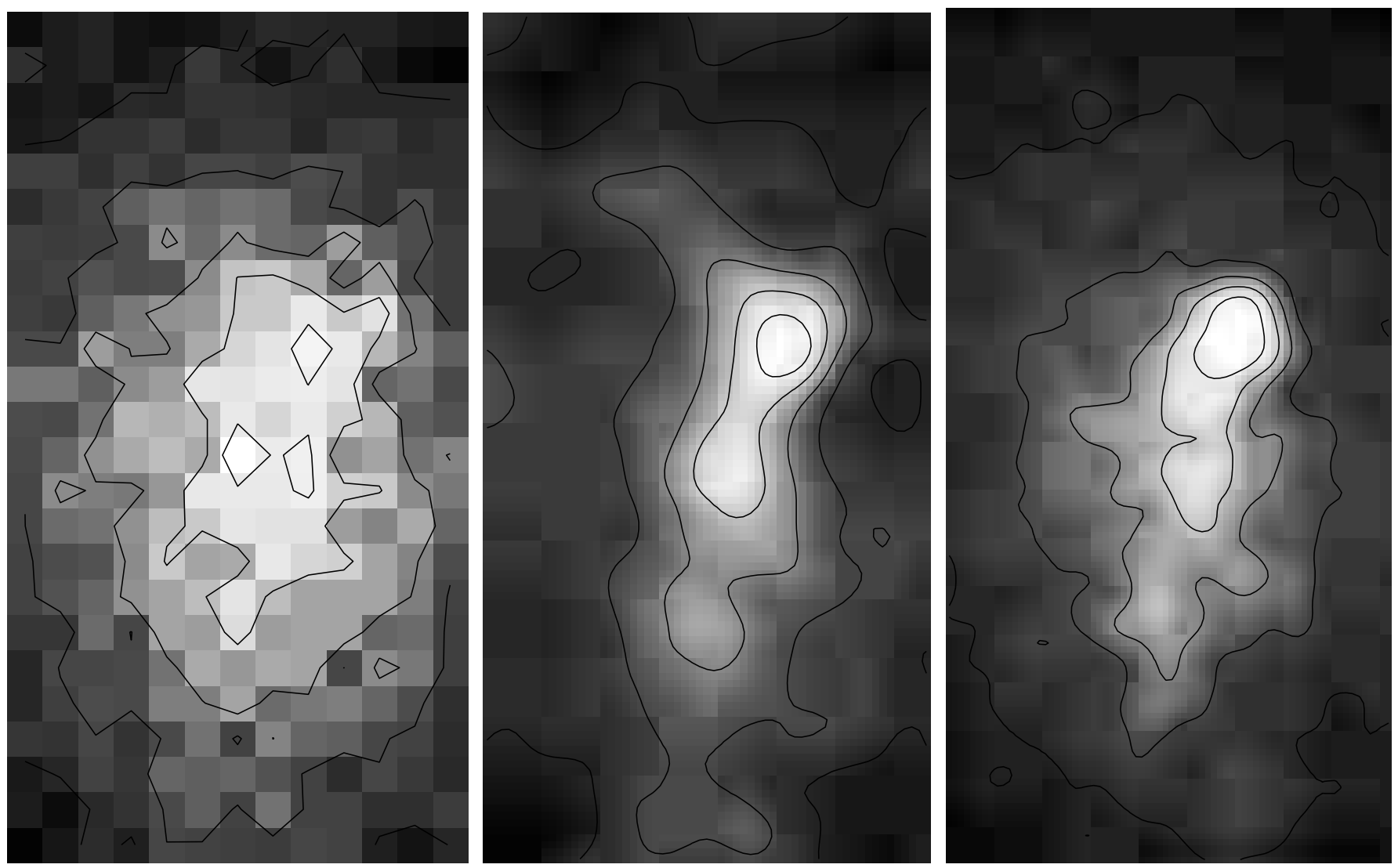

Fig. 2. Abell 1795 images. left: XMM pn raw image $(0.5 \sim 10 \mathrm{keV}$, pixel size $=2.5$ arcsec); center: restored image from left (pixel size $=0.5$ arcsec); right: Chandra ACIS-I image smoothed by a Gaussian filter with the $\sigma$ of 1.2 arcsec (whole energy band).

XMM (Böhringer et al. 2001). The spectral fitting parameters in the above two references are compared with our results in Table 2. The power-law indices we derived are consistent to within 2 3 standard deviations with previous results; the measured flux deficit of several percents with XMM pn at higher energies compared to XMM MOS (http://xmm.vilspa.esa.nl/docs/documents/CAL-TNQQ23-1-Q-ps.gz) may also contribute to the softer XMM reconstructed spectra. The differences on the power-law normalization factors may be due to the combination of two factors: (1) the relative normalization of the two instruments is not certain and; (2) the fluctuation of the M 87 nucleus flux of about $40 \%$ between two observations (Wilson \& Yang 2002).

\section{Discussion}

The results presented above demonstrate that the spatial resolution of XMM EPIC images can be improved by the DD technique. Especially for EPIC pn, which have a very large effective area, we can reach higher spatial resolution images than before and use them to obtain good quality spectra. Comparing these three restored images with Chandra images, we find that the 

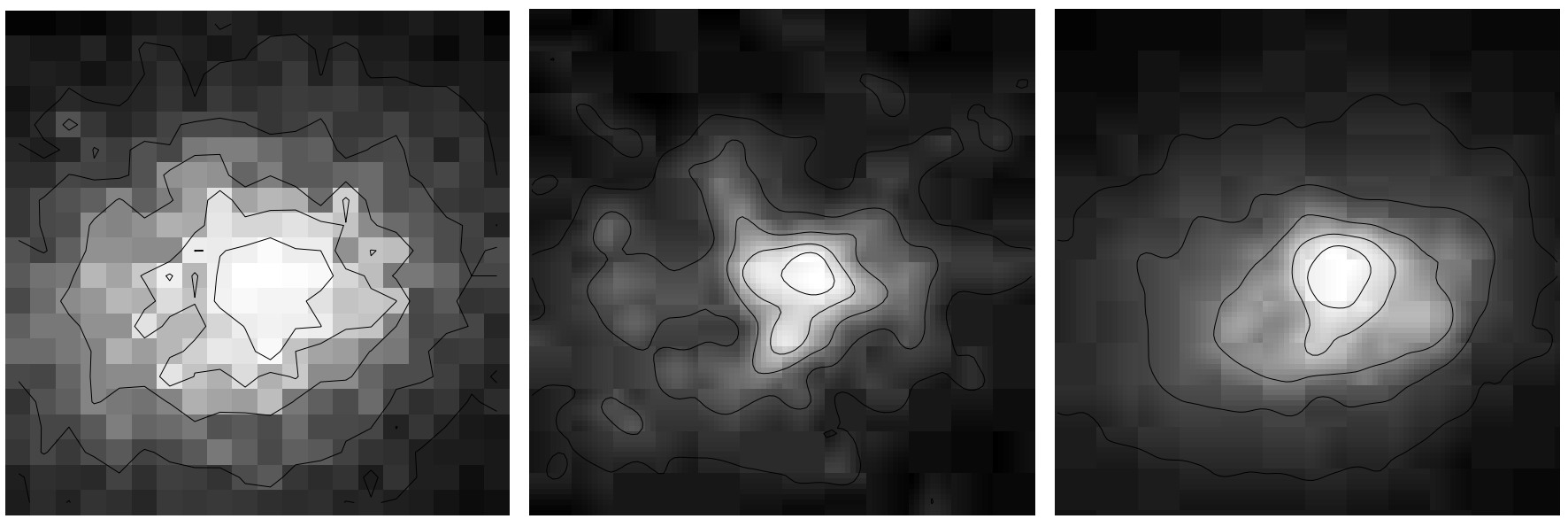

Fig. 3. PKS 0745-191 images. left: XMM pn raw image $(0.5 \sim 10 \mathrm{keV}$, pixel size $=2.5$ arcsec); center: restored image from left (pixel size $=0.5$ arcsec); right: Chandra ACIS-I image smoothed by a Gaussian filter with the $\sigma$ of 1.2 arcsec (whole energy band).
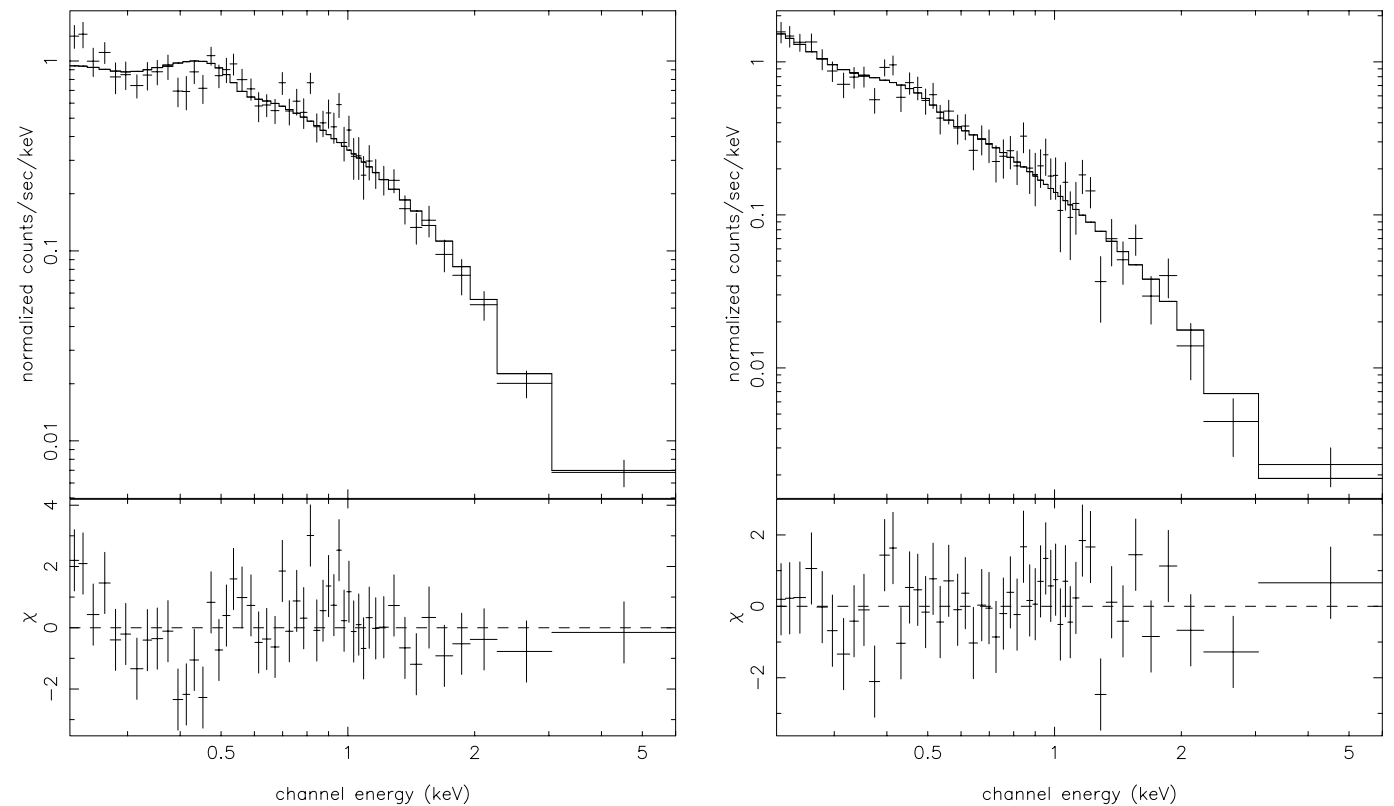

Fig. 4. Spectra of the nucleus (left) and the brighest knot (right) of M 87 from restored images $(0.2 \sim 6 \mathrm{keV})$.

Table 2. Comparisons of spectral parameters.

\begin{tabular}{lrcccc}
\hline \hline Name & $\begin{array}{r}\text { Region } \\
\text { (Radius) }\end{array}$ & $\begin{array}{c}n_{\mathrm{H}}^{\mathrm{a}, \mathrm{b}} \\
\left(10^{20} \mathrm{~cm}^{-2}\right)\end{array}$ & PhoIndex $^{\mathrm{c}}$ & norm $^{\mathrm{d}}$ & $\begin{array}{c}\chi^{2} \\
\text { (d.o.f.) }\end{array}$ \\
\hline Nucleus (Chandra) & $1.25^{\prime \prime}$ circ & $7.4_{-1.4}^{+1.5}$ & $2.25_{-0.09}^{+0.10}$ & $40.5_{-2.9}^{+2.4}$ & $145.2(123)$ \\
Knot (Chandra) & $1^{\prime \prime}$ circ & $1.3_{-1.1}^{+1.2}$ & $2.32_{-0.10}^{+0.11}$ & $20.9_{-1.1}^{+1.4}$ & $152.4(98)$ \\
Nucleus (XMM MOS) & $4^{\prime \prime}$ circ & N.A. & $2.25_{-0.06}^{+0.04}$ & N.A. & $170(178)$ \\
Knot (XMM MOS) & $3.5^{\prime \prime}$ circ & N.A. & $2.45_{-0.08}^{+0.11}$ & N.A. & $101(110)$ \\
Nucleus (XMM pn) & $5^{\prime \prime}$ circ & N.A. & $2.2_{-0.2}^{+0.2}$ & N.A. & N.A. \\
Knot (XMM pn) & $5^{\prime \prime}$ circ & N.A. & $2.5_{-0.4}^{+0.4}$ & N.A. & N.A. \\
Nucleus (Restored XMM pn) & $2.5^{\prime \prime}$ circ & $5.1_{-1.4}^{+1.7}$ & $2.48_{-0.13}^{+0.15}$ & $44.5_{-3.5}^{+4.1}$ & $65.4(47)$ \\
Knot (Restored XMM pn) & $2.5^{\prime \prime}$ circ & $0.2_{-0.2}^{+1.0}$ & $2.71_{-0.12}^{+0.18}$ & $16.4_{-1.3}^{+1.4}$ & $44.0(47)$ \\
\hline
\end{tabular}

a Equivalent hydrogen-absorbing column.

b All errors are $90 \%$ confidence for a signle parameter of interest.

c Photon index.

d Normalization of power-law model, in units of $10^{-5}$ photons $\mathrm{keV}^{-1} \mathrm{~cm}^{-2} \mathrm{~s}^{-1} @ 1 \mathrm{keV}$.

e Not avaliable in the reference paper. 
restored resolution is about $3 \operatorname{arcsec}(H E W)$. The only drawback is that the DD process introduces additional fluctuations to the spectral study. We find that the additional fluctuations depend on the total counts and their distribution in the source. In general, this effect is not serious for diffuse sources without sharp features, such as clusters of galaxies, supernova remnants, etc. The improvement in spatial resolution will reveal much more information about their structures, which usually leads to more precise and reliable spectral studies of structures.

Acknowledgements. We sincerely thank the anonymous referee, whose professionalism and constructive comments and suggestions have improved the work significantly. This study is supported in part by the Special Funds for Major State Basic Research Projects and by the National Natural Science Foundation of China. SNZ also acknowledges supports by NASA's Marshall Space Flight Center and through NASA's Long Term Space Astrophysics Program. We thank Dr. D. Grupe from MPE, Dr R. Starling from MSSL and XMM helpdesk@ vilspa for providing technical information about XMM and their helpful advices.

\section{References}

Böhringer, H., Belsole, E., Kennea, J., et al. 2001, A\&A, 365, L181

Chen, Y., Li, T. P., \& Wu, M. 1997, proceedings of ADASS VI, ed. G. Hunt, \& H. E. Payne, ASP Conf. Ser., 125, 178

Chen, Y., Li, T. P., \& Wu, M. 1998, A\&AS, 128, 363

Chen, Y., Song, L. M., Li, T. P., \& Cui, W. 2000, Acta Astron. Sinica, 41,214

Högbom, J. 1974, A\&AS, 15, 417

Li, T. P., \& Wu, M. 1994, Ap\&SS, 215, 21

Li, T. P. 1995, Exper. Astron., 6, 63

Li, T. P. 1997, Space observation of hard X-rays, in Space Science in China, ed. W. R. Hu (Gordon \& Breach Publisher)

Lu, F. J., Li, T. P., Sun, X. J., Wu, M., \& Page, C. G. 1996, A\&AS, 115,395

Lu, F. J., Li, T. P., Sun, X. J., \& Wu, M. 1996, in ed. R. Shellard, \& T. Nguyen, Proc. Int. Conf. on Computing in High Energy Physics (World Scintific Publishing Co.), 848

Lu, F. J., Aschenbach, B., \& Song, L. M. 2001, A\&A, 370, 570

Wilson, A. S., \& Yang, Y. 2002, ApJ, 568, 133

Zhang, S., Li, T. P., Wu, M., et al. 1997, AIP Conf. Proc., 410, 578

Zhang, S., Li, T. P., \& Wu, M. 1998, A\&A, 340, 62 